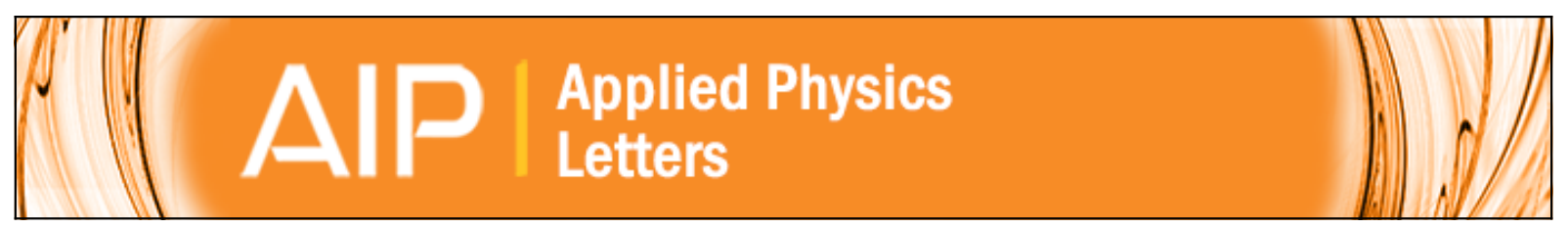

\title{
Transfer-free synthesis of highly ordered Ge nanowire arrays on glass substrates
}

M. Nakata, K. Toko, W. Jevasuwan, N. Fukata, N. Saitoh, N. Yoshizawa, and T. Suemasu

Citation: Applied Physics Letters 107, 133102 (2015); doi: 10.1063/1.4932054

View online: http://dx.doi.org/10.1063/1.4932054

View Table of Contents: http://scitation.aip.org/content/aip/journal/apl/107/13?ver=pdfcov

Published by the AIP Publishing

\section{Articles you may be interested in}

Growth strategies to control tapering in Ge nanowires

APL Mater. 2, 046105 (2014); $10.1063 / 1.4870875$

Controlled growth of SiGe nanowires by addition of $\mathrm{HCl}$ in the gas phase

J. Appl. Phys. 110, 024311 (2011); 10.1063/1.3610409

The growth and radial analysis of Si/Ge core-shell nanowires

Appl. Phys. Lett. 97, 251912 (2010); 10.1063/1.3531631

Band engineered epitaxial $\mathrm{Ge}-\mathrm{Si}$ x Ge 1 - x core-shell nanowire heterostructures

Appl. Phys. Lett. 95, 033101 (2009); 10.1063/1.3173811

The impacts of surface conditions on the vapor-liquid-solid growth of germanium nanowires on $\mathrm{Si}(100)$ substrate Appl. Phys. Lett. 93, 041917 (2008); 10.1063/1.2968201

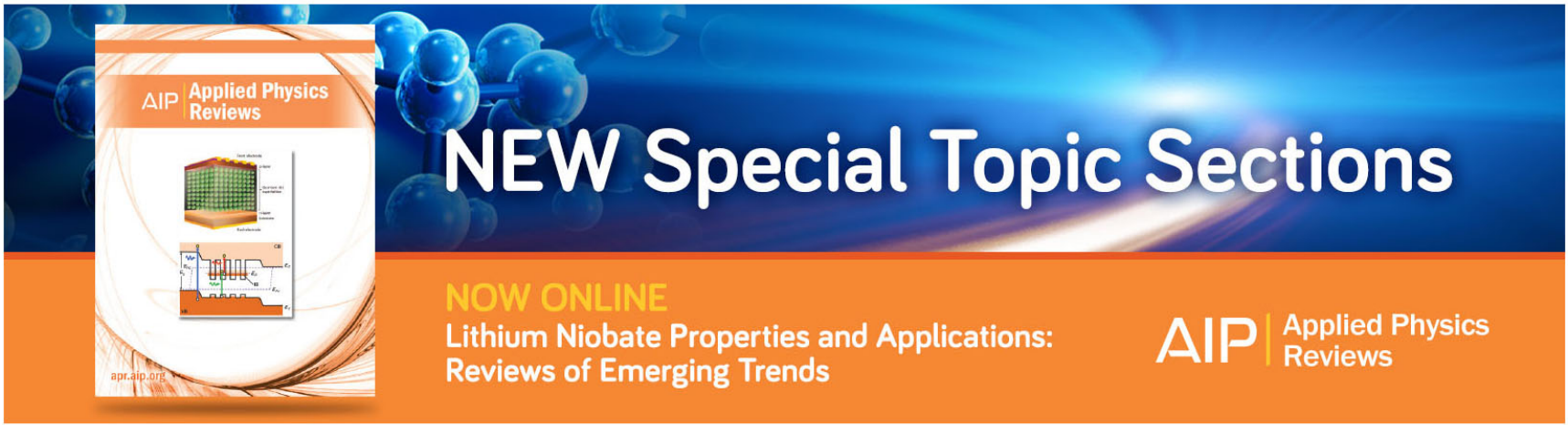




\title{
Transfer-free synthesis of highly ordered Ge nanowire arrays on glass substrates
}

\author{
M. Nakata, ${ }^{1}$ K. Toko,,${ }^{1, a)}$ W. Jevasuwan, ${ }^{2}$ N. Fukata, ${ }^{2}$ N. Saitoh, ${ }^{3}$ N. Yoshizawa, ${ }^{3}$ \\ and T. Suemasu ${ }^{1}$ \\ ${ }^{1}$ Institute of Applied Physics, University of Tsukuba, 1-1-1 Tennodai, Tsukuba, Ibaraki 305-8573, Japan \\ ${ }^{2}$ National Institute for Materials Science, 1-1 Namiki, Tsukuba 305-0044, Japan \\ ${ }^{3}$ Electron Microscope Facility, TIA, AIST, 16-1 Onogawa, Tsukuba 305-8569, Japan
}

(Received 7 July 2015; accepted 14 September 2015; published online 29 September 2015)

\begin{abstract}
Vertically aligned Ge nanowires (NWs) are directly synthesized on glass via vapor-liquid-solid (VLS) growth using chemical-vapor deposition. The use of the (111)-oriented Ge seed layer, formed by metal-induced crystallization at $325^{\circ} \mathrm{C}$, dramatically improved the density, uniformity, and crystal quality of Ge NWs. In particular, the VLS growth at $400{ }^{\circ} \mathrm{C}$ allowed us to simultaneously achieve the ordered morphology and high crystal quality of the Ge NW array. Transmission electron microscopy demonstrated that the resulting Ge NWs had no dislocations or stacking faults. Production of high-quality NW arrays on amorphous insulators will promote the widespread application of nanoscale devices. (C) 2015 AIP Publishing LLC. [http://dx.doi.org/10.1063/1.4932054]
\end{abstract}

There is wide interest in the development of semiconductor nanowires (NWs) because of their potential in scaling electronic optical devices. ${ }^{1-4}$ Ge NWs are particularly regarded as promising materials for a number of applications, including field-effect transistors, ${ }^{5}$ photodetectors, ${ }^{6}$ and lithium-ion batteries. ${ }^{7}$ Vapor-liquid-solid (VLS) growth, i.e., chemical vapor deposition (CVD) seeded by metal nanoparticles, is the method most frequently used to synthesize Ge NW arrays. ${ }^{8-17}$ To achieve ordered Ge NW arrays using the VLS method, (111)-oriented Si or Ge substrates are often used because Ge NWs grow in the $\langle 111\rangle$ direction when the diameter of the metal catalysts is larger than approximately $10 \mathrm{~nm} \cdot{ }^{10,11}$

NW arrays on amorphous insulators, such as $\mathrm{SiO}_{2}$ or glass, have gained much attention for further expanding the application of nanoscale devices. ${ }^{18-21}$ Transferring aligned NWs to amorphous substrates is a promising technique; ${ }^{20,21}$ however, there are difficulties keeping the process costs low and fabricating large-area devices, such as displays or solar cells. The transfer-free synthesis of NW arrays on amorphous substrates has been investigated for over a decade. For oxidized materials, such as $\mathrm{ZnO}$ and $\mathrm{TiO}_{2}$, hydrothermal methods have allowed for the direct synthesis of vertically aligned NW arrays on amorphous substrates. ${ }^{22,23}$ On the other hand, single-element nanowires, including Ge, have never been ordered on amorphous substrates because the growth occurred in random directions. ${ }^{24,25}$ To control the growth direction of Ge NWs on amorphous substrates, here we propose a way using an orientation-controlled seed layer formed by metal-induced crystallization. ${ }^{26-30}$ The highly ordered Ge NW array is directly synthesized on a glass substrate, which will lead to advanced electronic and optical devices with low-cost substrates.

Figure 1(a) schematically shows the preparation process of the sample. Al-induced crystallization (AIC) was used to form a (111)-oriented Ge seed layer. An Al layer (thickness:

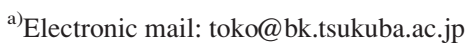

$50 \mathrm{~nm}$ ) was prepared and then exposed to air for $10 \mathrm{~min}$ to form a native $\mathrm{AlO}_{\mathrm{x}}$ membrane, followed by preparing an amorphous Ge layer (thickness: $50 \mathrm{~nm}$ ). The depositions were carried out using a radio frequency magnetron sputtering with Ar plasma. After that, the sample was annealed at $325^{\circ} \mathrm{C}$ for $100 \mathrm{~h}$ in $\mathrm{N}_{2}$, which crystallized Ge via exchange between $\mathrm{Ge}$ and $\mathrm{Al}$ layers. ${ }^{28,29}$ The $\mathrm{Al}$ layer and the $\mathrm{Ge}$ islands, remaining in the top layer, were removed using $\mathrm{HF}$ (HF: $1.5 \%$ ) and $\mathrm{H}_{2} \mathrm{O}_{2}$ (HF: $50 \%$ ) solutions. ${ }^{30}$ This resulted

(a)
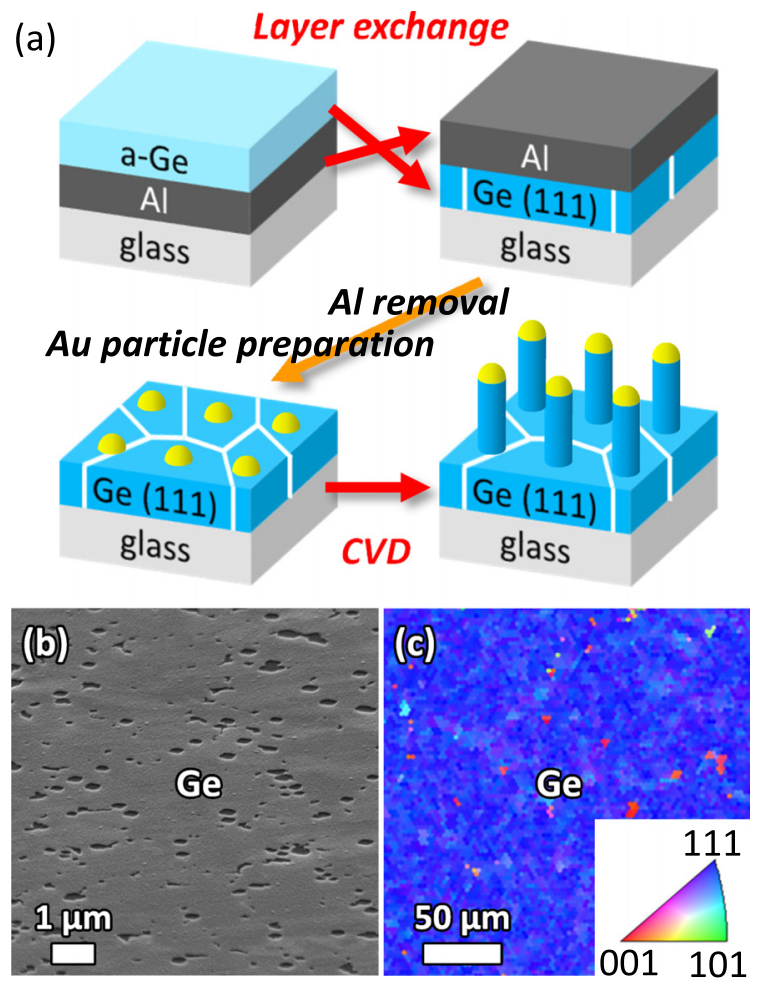

FIG. 1. (a) Schematic of the preparation procedure of the sample with a Ge seed layer. (b) SEM image of the surface of a Ge seed layer, where the sample is $70^{\circ}$ tilted. (c) EBSD image of a Ge seed layer, where the coloration indicates crystal orientation, according to the legend. 


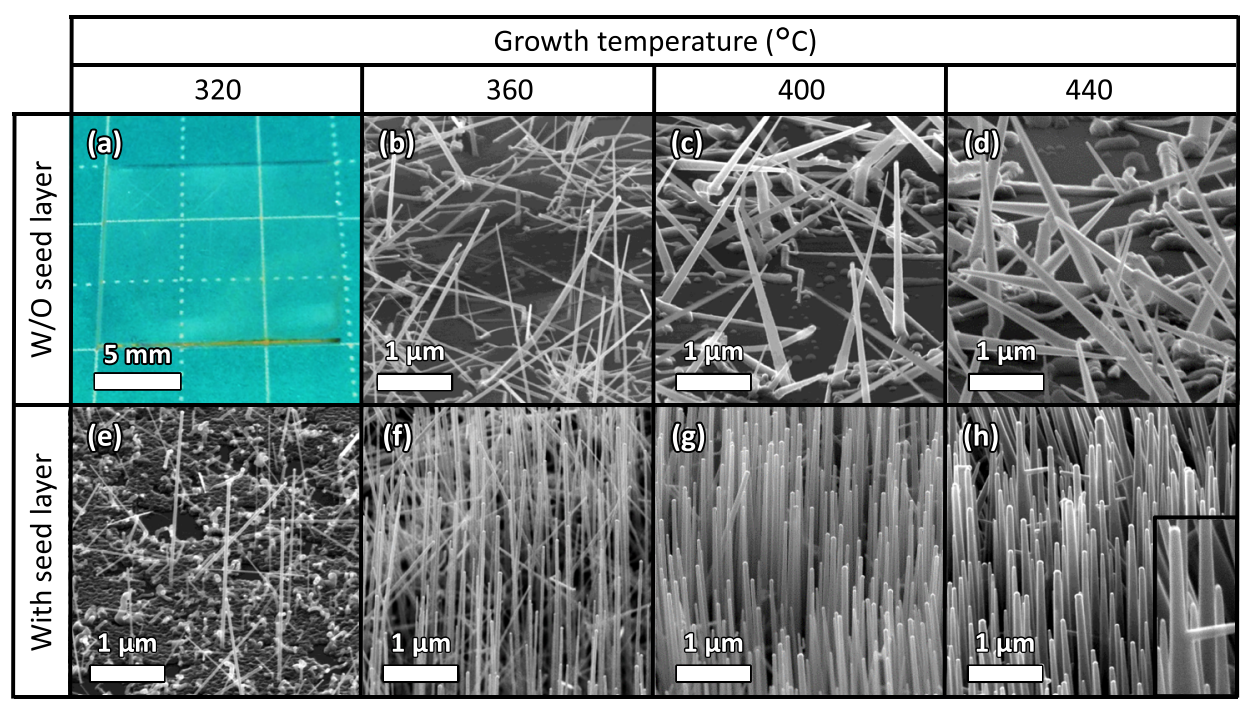

FIG. 2. Photograph and SEM images of the samples, (a)-(d) without and (e)-(h) with a Ge seed layer, after CVD at $320^{\circ} \mathrm{C}, 360^{\circ} \mathrm{C}, 400^{\circ} \mathrm{C}$, and $440^{\circ} \mathrm{C}$. The inset in (h) shows a magnified image indicating a taper and a branch. In the SEM, the samples are $70^{\circ}$ tilted.

in the uniform surface of the Ge layer as shown in the scanning electron microscopy (SEM) image of Fig. 1(b). An electron backscatter diffraction (EBSD) image of Fig. 1(c) indicates that the Ge layer is highly (111)-oriented. After preparing the (111)-oriented Ge seed layer, VLS growth was performed as follows. Au nanocolloid particles (diameter: $40 \mathrm{~nm}$ ) were prepared on the surface by dipping the sample in a colloidal solution. After that, CVD using $10 \mathrm{sccm} \mathrm{GeH}_{4}$ $(100 \%)$ was performed at $320-440{ }^{\circ} \mathrm{C}$ for $10 \mathrm{~min}$. For comparison, the VLS growth was also performed using a bare glass substrate.

The VLS growth morphology was evaluated by SEM. Figure 2 shows the results of the samples grown at $320^{\circ} \mathrm{C}$, $360{ }^{\circ} \mathrm{C}, 400^{\circ} \mathrm{C}$, and $440{ }^{\circ} \mathrm{C}$. The $320^{\circ} \mathrm{C}$ grown sample without a seed layer was charged up by the electrons on the insulating substrate during SEM observation, because the sample has few NWs, as seen in the photograph of Fig. 2(a). Figures 2(b)-2(d) show that the growth temperatures over $360^{\circ} \mathrm{C}$ allow Ge NWs to be formed on a glass substrate. However, the Ge NW arrays are low-density and disordered. This may have occurred because the substrate is amorphous and because it is difficult for Ge atoms to adhere to $\mathrm{SiO}_{2}$ during CVD. ${ }^{25,31}$

Figures 2(e)-2(h) show that the use of the AIC-Ge seed layer significantly improves the VLS growth morphology. In particular, when the growth temperatures are over $400{ }^{\circ} \mathrm{C}$, high-density, vertically aligned Ge NW arrays are formed. These results suggest that the Ge NWs were seeded by the (111)-oriented Ge layer and grew in the [111] direction. Thus, the AIC-Ge seed layer has two roles: enhancing the nucleation frequency and controlling the growth direction of Ge NWs. Note that a temperature of $440^{\circ} \mathrm{C}$ is too high because it causes the branching of NWs as shown in Fig. 2(h).

The crystal quality of Ge NWs was evaluated by microprobe Raman scattering spectroscopy (spot size: $1 \mu \mathrm{m}$, wavelength: $532 \mathrm{~nm}$ ). Figure 3(a) shows that all samples with a Ge seed layer have peaks at approximately $300 \mathrm{~cm}^{-1}$, corresponding to the Ge-Ge vibration mode. ${ }^{17}$ According to our previous study, the Ge-Ge peaks of 50-nm-thick AIC-Ge layers are relatively broad and present at approximately $292 \mathrm{~cm}^{-1}$ likely because of the residual $\mathrm{Al}$ atoms in Ge. ${ }^{29}$
The Ge-Ge peaks in Fig. 3(a), therefore, are considered to be attributed to Ge NWs. Figure 3(b) shows that the peak positions of the $\mathrm{Ge}-\mathrm{Ge}$ peaks remain unchanged regardless of different growth temperatures and are the same as that of a bulk Ge substrate. On the other hand, the full width of half maximums (FWHMs) of the Ge-Ge peaks decrease with increasing the growth temperature and approach that of a bulk-Ge substrate at temperatures over $400^{\circ} \mathrm{C}$. Considering the SEM and Raman studies, $400{ }^{\circ} \mathrm{C}$ is almost the optimum growth temperature for simultaneously achieving the ordered morphology and high crystal quality of Ge NWs.

We investigated the effect of the Ge seed layer on the crystal quality of Ge NWs. Figure 3(c) shows that the Ge-Ge peak of the sample with the Ge seed layer has a larger
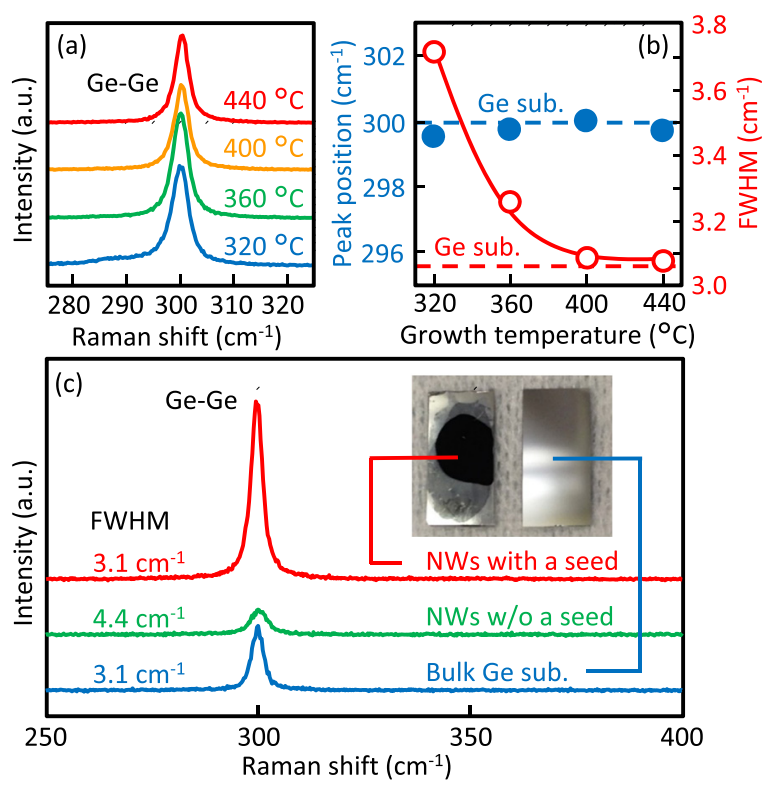

FIG. 3. (a) Raman spectra of the samples with a Ge seed layer, where the CVD temperatures are $320^{\circ} \mathrm{C}, 360^{\circ} \mathrm{C}, 400^{\circ} \mathrm{C}$, and $440{ }^{\circ} \mathrm{C}$. (b) Growth temperature dependence of the peak positions (closed circles) and FWHMs (open circles) of the Ge-Ge peaks in (a). The data of the Ge-Ge peak for a bulk-Ge wafer are shown by dotted lines. (c) Raman spectra of $400{ }^{\circ} \mathrm{C}$ grown samples with and without a Ge seed layer, and those of a bulk-Ge wafer. FWHMs of the Ge-Ge peaks are shown on the left side. The inset shows a photograph of the samples, indicating that the Ge NW array, corresponding to the dark area, is antireflective. 
intensity and a smaller FWHM compared to that of the sample without the Ge seed layer. This result indicates that the use of the Ge seed layer improves not only the density but also the crystal quality of Ge NWs. Note that the peak intensity of the sample with the Ge seed layer is even larger than that of a bulk-Ge substrate, which is attributed to the antireflection effect of the NW structure, as shown in the inset of Fig. 3(c). The antireflection effect is an advantage of the NW structure for fabricating high-efficiency optical devices. ${ }^{1-3}$

To evaluate the detailed crystal structure of Ge NWs grown on a $\mathrm{Ge}$ seed layer at $400{ }^{\circ} \mathrm{C}$, we used the analytical transmission electron microscopy (TEM), FEI Tecnai Osiris operated at $200 \mathrm{kV}$, equipped with an energy dispersive Xray spectrometer (EDX) and a scanning transmission electron microscopy (STEM) system with a probe diameter of $\sim 1 \mathrm{~nm}$. The representative results among several NWs are shown in Figs. 4 and 5. Figures 4(a)-4(c) indicate that the Ge NW, $3.2 \mu \mathrm{m}$ in length, is tapered. The Ge NWs had the dispersion of their head diameters, 30-60 nm, likely attributed to the diameter dispersion of the initial Au particles. The bottom diameters of the Ge NWs were approximately $100 \mathrm{~nm}$ larger than their head diameters. Because the nanowire tapering is caused by vapor-solid growth on the sidewall surfaces of NWs, it can be avoided in needed by controlling the CVD growth step. ${ }^{11,17}$ The selected-area electron diffraction (SAED) pattern shown in Fig. 4(d) proves that the Ge NW is [111]-oriented in the growth direction.

Figure 5(a) obtained by STEM-EDX analysis shows that a round-shape Au particle is present on a Ge NW head, which is evidence of VLS growth. The Ge surface is covered by natively oxidized $\mathrm{Ge}\left(\mathrm{GeO}_{x}\right)$ membranes. Figure 5(b) indicates that the NW head is pure Au while the NW bottom is pure Ge. The metal contaminations in the Ge NWs, possibly coming from catalytic Au particles or residual $\mathrm{Al}$ in the seed layer, were below the EDX detection limit $(\sim 1 \%)$. Figure 5(c) shows a uniform bright contrast of the Ge NW,

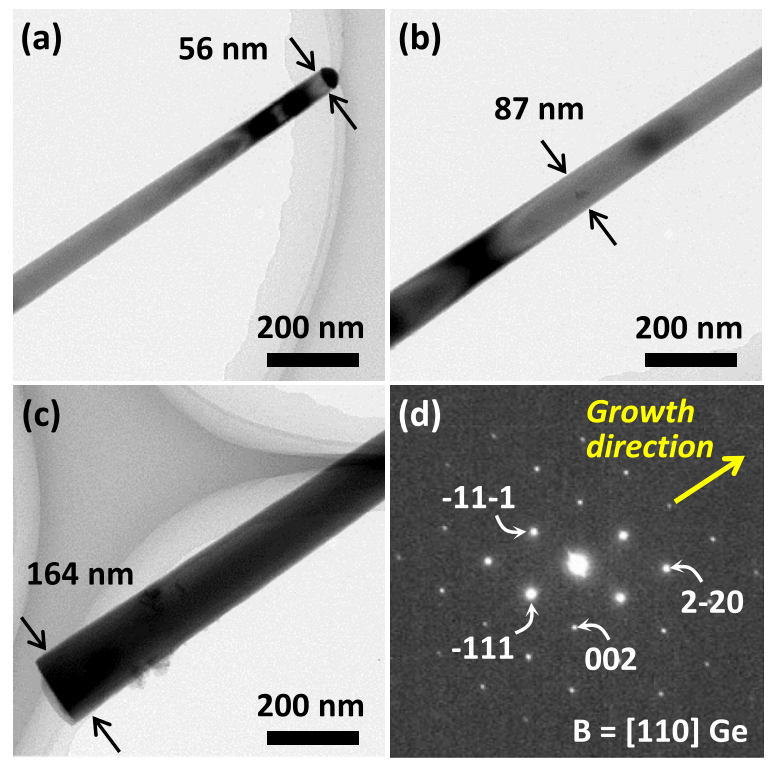

FIG. 4. (a)-(c) Bright-field TEM images of a Ge $\mathrm{NW}$ in a $400^{\circ} \mathrm{C}$ grown sample with a Ge seed layer, showing its (a) head, (b) body, and (c) bottom. (d) SAED pattern in Ge $\langle 110\rangle$ zone axis taken from the Ge NW shown in (a)-(c).


FIG. 5. Detailed structures of a Ge NW in a $400^{\circ} \mathrm{C}$ grown sample with a $\mathrm{Ge}$ seed layer. (a) EDX elemental map of its head. (b) EDX spectra of its head and bottom, where $\mathrm{Cu}$ signals are attributed to the sample stage. (c) and (d) Dark-field TEM images with magnification of (c) $6300 \times$ and (d) $64000 \times$, using the Ge $\{111\}$ plane reflection. (e) High-resolution TEM image in $\mathrm{Ge}\langle 110\rangle$ zone axis taken around the sidewall of the Ge NW.

indicating single crystalline Ge with no grain boundaries. As representatively shown in Fig. 5(d), the Ge NWs had no dislocations or stacking faults in the entire region. Figure 5(e) shows clear (111) lattice fringes, indicating the high crystallinity of the Ge NWs as well as the growth in [111] direction.

In conclusion, we fabricated a vertically aligned, defectfree Ge NW array on a glass substrate using a transfer-free process. To control the growth direction of Ge NWs during VLS growth, a (111)-oriented Ge seed layer (thickness: $50 \mathrm{~nm}$ ) was prepared using AIC at $325^{\circ} \mathrm{C}$. The use of the seed layer dramatically improved both the growth morphology and the crystal quality of Ge NWs, which was particularly prominent in the VLS growth at $400^{\circ} \mathrm{C}$. TEM observation demonstrated that the resulting Ge NWs were single crystalline and even free from obvious defects such as dislocations or stacking faults. This achievement holds promise for promoting the widespread application of NWbased electronic and optical devices.

This work was financially supported by the JSPS KAKENHI (Nos. 26709019 and 26600083) and the JGC-S Scholarship Foundation. Some experiments were conducted at the International Center for Young Scientists in NIMS.

${ }^{1}$ Y. Li, R. Clady, J. Park, S. V. Thombare, T. W. Schmidt, M. L. Brongersma, and P. C. McIntyre, Nano Lett. 14, 3427 (2014).

${ }^{2}$ B. Polyakov, B. Daly, J. Prikulis, V. Lisauskas, B. Vengalis, M. A. Morris,

J. D. Holmes, and D. Erts, Adv. Mater. 18, 1812 (2006).

${ }^{3}$ A. Solanki and K. Crozier, Appl. Phys. Lett. 105, 191115 (2014). 
${ }^{4}$ K. Tomioka, M. Yoshimura, and T. Fukui, Nature 488, 189 (2012).

${ }^{5}$ J. Xiang, W. Lu, Y. Hu, Y. Wu, H. Yan, and C. M. Lieber, Nature 441, 489 (2006).

${ }^{6}$ C.-J. Kim, H.-S. Lee, Y.-J. Cho, K. Kang, and M.-H. Jo, Nano Lett. 10, 2043 (2010).

${ }^{7}$ J. Wang, N. Du, H. Zhang, J. Yu, and D. Yang, J. Mater. Chem. 22, 1511 (2012).

${ }^{8}$ C. O'Regan, S. Biswas, N. Petkov, and J. D. Holmes, J. Mater. Chem. C 2, 14 (2014)

${ }^{9}$ H. Adhikari, A. F. Marshall, I. A. Goldthorpe, C. E. D. Chidsey, and P. C. McIntyre, ACS Nano 1, 415 (2007).

${ }^{10}$ S. A. Dayeh and S. T. Picraux, Nano Lett. 10, 4032 (2010).

${ }^{11}$ H. Adhikari, A. F. Marshall, C. E. D. Chidsey, and P. C. McIntyre, Nano Lett. 6, 318 (2006).

${ }^{12}$ S. Kodambaka, J. Tersoff, M. C. Reuter, and F. M. Ross, Science 316, 729 (2007).

${ }^{13}$ J. H. Woodruff, J. B. Ratchford, I. A. Goldthorpe, P. C. McIntyre, and C. E. D. Chidsey, Nano Lett. 7, 1637 (2007).

${ }^{14}$ Y. Sierra-Sastre, S. Choi, S. T. Picraux, and C. A. Batt, J. Am. Chem. Soc. 130, 10488 (2008).

${ }^{15}$ M. Koto, A. F. Marshall, I. A. Goldthorpe, and P. C. McIntyre, Small 6, 1032 (2010).

${ }^{16}$ J. G. Connell, Z. Y. Al Balushi, K. Sohn, J. Huang, and L. J. Lauhon, J. Phys. Chem. Lett. 1, 3360 (2010).

${ }^{17}$ N. Fukata, K. Sato, M. Mitome, Y. Bando, T. Sekiguchi, M. Kirkham, J.-I. Hong, Z. L. Wang, and R. L. Snyder, ACS Nano 4, 3807 (2010); N. Fukata, M. Mitome, T. Sekiguchi, Y. Bando, M. Kirkham, J.-I. Hong, Z. L. Wang, and R. L. Snyder, ibid. 6, 8887 (2012).
${ }^{18}$ W. Y. Weng, S. J. Chang, C. L. Hsu, and T. J. Hsueh, ACS Appl. Mater. Interfaces 3, 162 (2011)

${ }^{19}$ S. W. Schmitt, F. Schechtel, D. Amkreutz, M. Bashouti, S. K. Srivastava, B. Hoffmann, C. Dieker, E. Spiecker, B. Rech, and S. H. Christiansen, Nano Lett. 12, 4050 (2012).

${ }^{20}$ J. Tang, C. Y. Wang, F. Xiu, M. Lang, L. W. Chu, C. J. Tsai, Y. L. Chueh, L. J. Chen, and K. L. Wang, ACS Nano 5, 6008 (2011).

${ }^{21}$ J. S. Huang, C. Y. Hsiao, S. J. Syu, J. J. Chao, and C. F. Lin, Sol. Energy Mater. Sol. Cells 93, 621 (2009).

${ }^{22}$ J. Liu, W. Wu, S. Bai, and Y. Qin, ACS Appl. Mater. Interfaces 3, 4197 (2011).

${ }^{23}$ X. Feng, K. Shankar, O. K. Varghese, M. Paulose, T. J. Latempa, and C. A. Grimes, Nano Lett. 8, 3781 (2008).

${ }^{24}$ D. Wang and H. Dai, Angew. Chem. 114, 4977 (2002).

${ }^{25}$ X. Sun, G. Calebotta, B. Yu, G. Selvaduray, and M. Meyyappan, J. Vac. Sci. Technol., B 25, 415 (2007).

${ }^{26}$ Z. Wang, L. Gu, L. P. H. Jeurgens, F. Phillipp, and E. J. Mittemeijer, Nano Lett. 12, 6126 (2012).

${ }^{27}$ M. Kurosawa, T. Sadoh, and M. Miyao, J. Appl. Phys. 116, 173510 (2014).

${ }^{28}$ K. Toko, M. Kurosawa, N. Saitoh, N. Yoshizawa, N. Usami, M. Miyao, and T. Suemasu, Appl. Phys. Lett. 101, 072106 (2012).

${ }^{29}$ K. Toko, R. Numata, N. Oya, N. Fukata, N. Usami, and T. Suemasu, Appl. Phys. Lett. 104, 022106 (2014).

${ }^{30}$ K. Toko, K. Nakazawa, N. Saitoh, N. Yoshizawa, and T. Suemasu, Cryst. Growth Des. 15, 1535 (2015).

${ }^{31}$ M. Tada, J.-H. Park, D. Kuzum, G. Thareja, J. R. Jain, Y. Nishi, and K. C. Saraswat, J. Electrochem. Soc. 157, H371 (2010). 